\title{
OPTOELECTRONIC MEASUREMENT OF INTERFACIAL SURFACE
}

\author{
K. Czernek ${ }^{*}$, S. Witczak ${ }^{* *}$
}

\begin{abstract}
The study presents the possible use of optoelectronic system for the measurement of the values which are specific for hydrodynamics of two-phase gas-liquid flow in vertical pipes, where a very-highviscosity liquid forms a falling film in a pipe. The experimental method was provided, and the findings were presented and analyzed for selected values which characterize the two-phase flow. Attempt was also made to evaluate the effects of flow parameters and properties of the liquid on the gas-liquid interface surface value, which is decisive for the conditions of heat exchange and mass transfer in falling film equipment.
\end{abstract}

Keywords: optoelectronic measurement system, very viscous liquid, two-phase flow

\section{Introduction}

Multi-phase flow is a common phenomenon in numerous engineering installations in industry and in various types of apparatus. The concurrent occurrence of gas and very viscous liquid phases in such systems result in many impediments in ensuring adequate operating conditions for apparatus in which we have to do with the processes of the heat, momentum and mass transfer (such as heat exchangers, evaporators, reactors). The various process conditions that correspond to each structures thereby constitute the basic obstacle in ensuring adequate flow regimes and, concurrently, maintenance of required process parameters along the entire flow path. Important role is attributed to such solutions that secure the optimum operation of the process apparatus within maximum time ranges, which has to accompanied by the elimination of adverse phenomena that can be associated with the course of the processes. The information found in the literature of the subject indicate that annular two-phase gas-liquid flow is applied more and more commonly, due to its efficiency with regard to processes of heat and mass transfer with the concurrent possibility of applying in these processes substances that vary in terms of physical properties.

Thin-film evaporators, film heat exchangers, film absorbers and thin-film condenser apparatus offer examples of apparatus that apply annular two-phase flow. To this date a large number of papers (Czernek et al., 2013, Wojtan et al., 2005, Xu, 2007) have been disseminated in description of almost all types of flow patterns in multi-phase flow. The research covered in these papers includes the issues pertaining to identification and description of the forming flow patterns, areas of their occurrence (flow maps), determination of pressure drop and volume fraction of the specific components of the mixture as well as thickness of fluid films and characteristics of their wavy structures - all of which are standard issues put forward in the description of hydrodynamics of two-phase gas-liquid flow. However, regardless of the means in which two-phase gas-liquid flow is formed, in flow channels we have to do with various structures that originate as a consequence of the mutual configuration of the phases. The form and type of such structures are relative to volumetric flux of each phases, geometry of the channel, its configuration (vertical, horizontal, other layouts) as well as the physical and chemical properties of the components of two-phase mixture, and in particular that of the liquid phase. These remarks are based on original

\footnotetext{
Assoc. Prof. Krystian Czernek, PhD.: Department of Process Engineering, Opole University of Technology, Mikolajczyka Street 5; 45-271, Opole; PL, k.czernek@po.opole.pl

** Prof. Stanisław Witczak, PhD.: Department of Process Engineering, Opole University of Technology, Mikolajczyka Street 5; 45-271, Opole; PL, s.witczak@po.opole.pl
} 


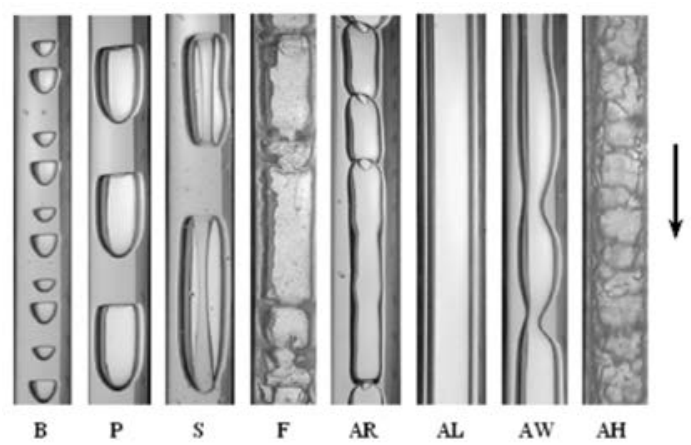

Fig. 1: Flow patterns of two-phase gas-very viscous liquid flow: $B$ - bubbly flow, $P$ - plug flow, $S$ - stalactite flow, $F$ - foam flow, $A R$ - annular core flow, $A L$ - annular smooth flow, $A W$ annular wave flow, $A H$ - annular hydraulic flow.

experimental research by these authors (Czernek et al., 2013 and Troniewski et al., 2006) conducted within liquid viscosity range up to $3500 \mathrm{mPa} \cdot \mathrm{s}$, an example of which is found in Fig. 1.

The classification of flow patterns is usually undertaken on the basis of visual observation. For the cases when such observation is impossible, various visual systems are applied, or unconventional observation techniques are applied, including: special photography systems (Czernek et al., 2013 and Troniewski et al., 2006), single- or multi-areas image capture in the cross-section of the stream (Czernek, 2013), photography by means of a beam of X rays (Stahl et al., 2004), optoelectronic cross-section image structure detectors (Oriol et al., 2008), holographic filming (Lee et al., 1986).

From the process perspective, such a course of research is well justified and even intentional since it can offer adequately high process results (increase of efficiency of heat exchange, improvement of flow rate, etc.). The areas of the occurrence of specific forms of two-phase flow are usually presented in charts that are called flow maps. They form a graphical interpretation of the flow conditions, through indication of the areas of the occurrence of the particular flow patterns that are separated into boundary lines in such maps.

A very relevant element that affects the course of heat and mass transfer process is the one connected with the surface of the exchange. For the co-current downward gas-very viscous liquid flow there is a possibility of disturbance in the inter-facial surface in the form of waves. Such disturbance increases the surface of the contact between the phases. The knowledge of the flow parameters, for which the contact surface is possibly the largest and has no influence on the reduction of the flow rate or other complication, offers the possibility of conducting the process in an apparatus with virtually too small interfacial surface.

\section{Methods}

The development of engineering methods and improvements in measurement devices have made it possible to gain results that had been otherwise impossible previously. Optoelectronic devices form an example of a measurement system that is applied in the realization of experiments. The linear measurement system was applied (Fig. 2), which consisted of four single-axis measurement sensors situated along the cross section of the channel, which applied the principle of absorption of light beam passing through an oil film. Whereas the point based system of optical probes made it possible to capture the linear state of the configuration of gas-liquid flux, the other applied system - multi-channel system of object-oriented image analysis - enabled the authors to assess the state with regard to the entire crosssection of the channel. In its principle, the system is formed by an optoelectronic image registration and analysis system based on a similar principle as a point-based one. However, in contrast to it, in this case a beam of light focused on the entire cross-section of the channel was applied and the image was captured by means of an endoscope digital camera situated in the axis of the measuring channel. Overall, this system is based on the principle of a laser knife, which involves registration of image that is visible in a vertical light beam that is focused in the cross-sectional plane of the channel with adjusting of the image resulting from the application of an optical system. The application of optoelectronic measuring system in the form of laser knife offered supplementary output to the optoelectronic linear system and enabled authors to extend the range of identification of geometrical parameters that are specific for the downward flow of liquid film. 

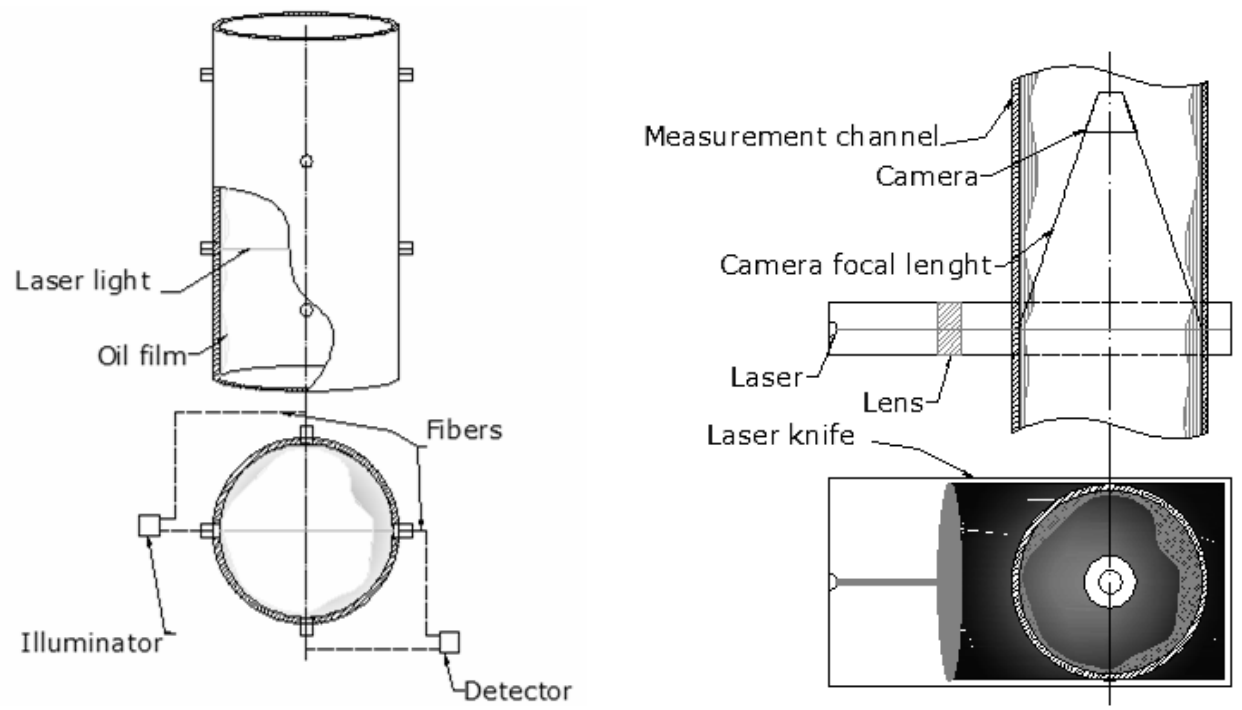

Fig. 2: Optoelectronic single-axis probe and diagram illustrating the operating principle of laser knife

In order to illustrate the changes occurring on the surface of the liquid film as a result of the increase of the velocity of co-current gas phase, Fig. 3 presents examples of planar images of this surface captured over the total circumference of the channel. These images concurrently reflect annular flow patterns and in quantitative framework - they indicate the change in downward liquid film referred to local conditions.

As one can note, an increase in the velocity of gas phase results in the occurrence of increasingly higher waves on the surface of liquid film. Beside the smooth and light wavy structures, they often have irregular character and the appearance of wavy states on the boundaries between phases have spatially and temporally variable amplitude and length. The spectrum of these changes indicates that regardless of the initially smooth liquid films, within the Reynolds numbers (4000 (laminar flow)-10000 (turbulent flow))
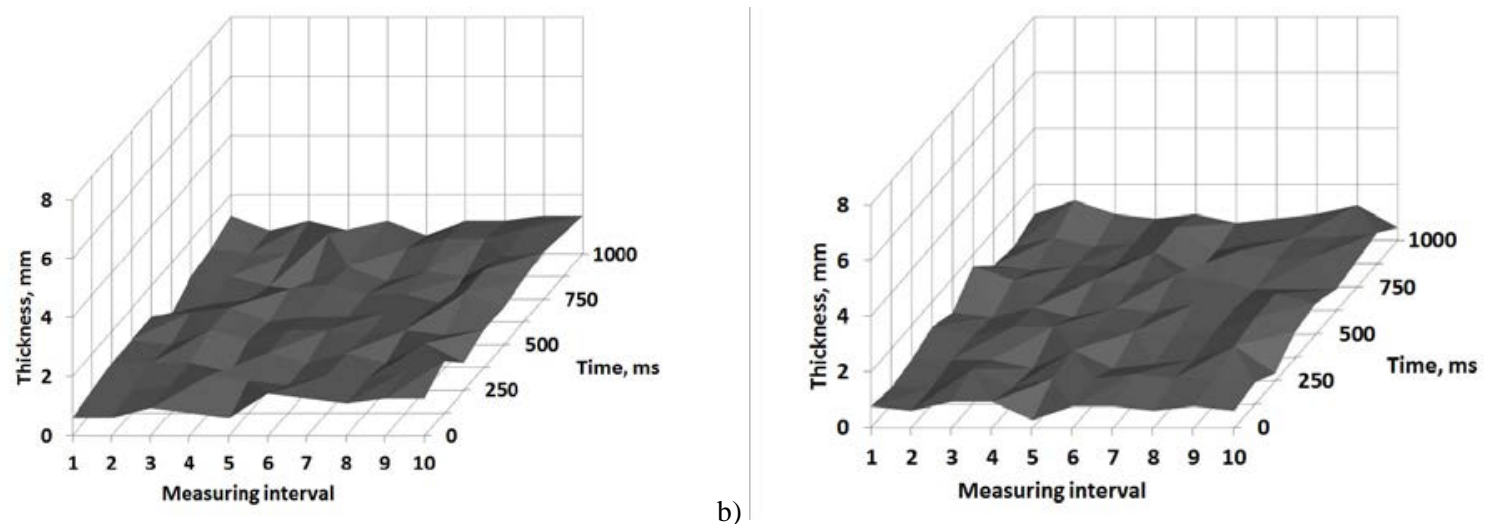

a)

b)
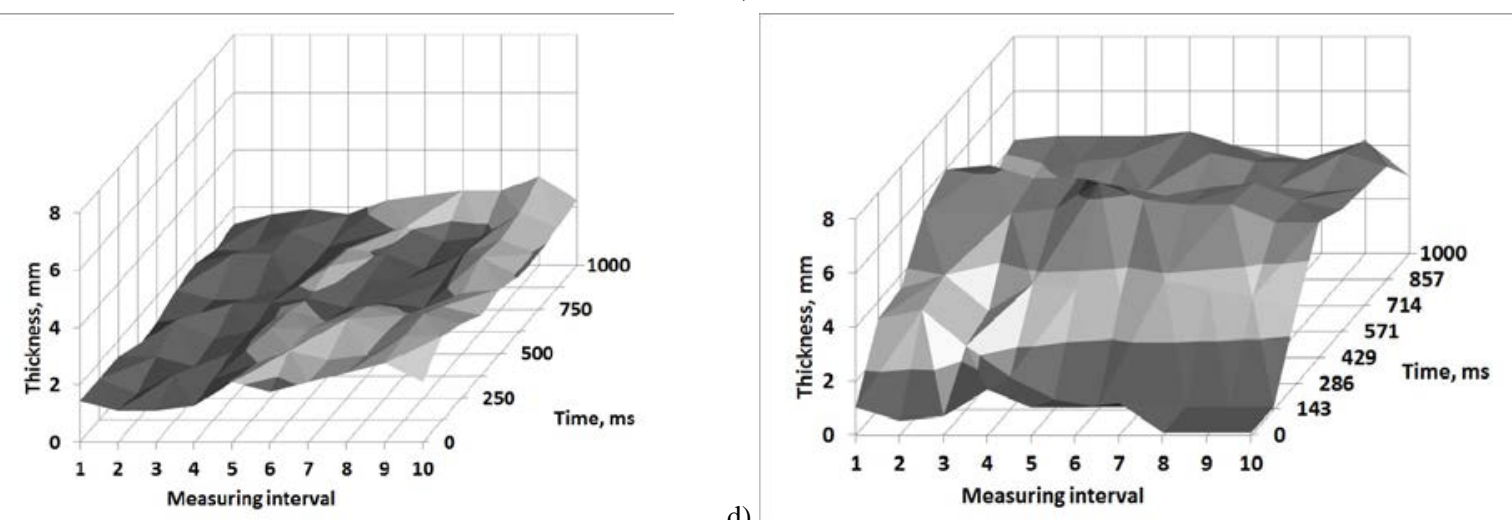

Fig. 3: Development of interfacial surface and distribution of oil film thickness in pipe:

a) smooth flow $\left(\operatorname{Re}_{g},=, 1300\right)$, b) light wavy flow $\left.\left(\operatorname{Re}_{g},=, 2250\right), c\right)$ wavy flow $\left(\operatorname{Re}_{g},=, 7800\right)$,

d) hydraulic - high waves $\left(\operatorname{Re}_{g},=, 14300\right)$. 
of the gas there will always be wavy structures and the nature of the development of waves and their dimensions are considerably relative to the viscosity of the liquid (the more viscous liquid, the larger wave damping).

Characteristics of two-phase gas-very viscous liquid flow form a considerably complex hydrodynamic phenomenon, which is accompanied by multidimensional asymmetry of the liquid film profile. This is further accompanied by stochastic characteristics of flow phenomena, whose scale is varied depending on the flow conditions. This is particularly discernible within the range of very wavy and hydraulic flow patterns, in which we have to do with very variable in time values of liquid film thickness. As a consequence, there is an occurrence of variable volume fraction of phases in the flow. In addition, some role is played by the variable interfacial surface in these conditions.

\section{Conclusions}

As a result of the analysis of the identified annular flow patterns of very viscous liquid flow it was discovered that depending on the viscosity of the liquid and mutual relations of velocities of the two phase gas-liquid the annular flow patterns very extremely - from a smooth liquid film until a highly dispersed hydraulic form.

The presented optoelectronic measurement systems made it possible to identify such flow parameters as: liquid film thickness, velocity of waves dispersion on the surface as well as their dynamics.

The identification and assessment of the flow phenomena of the annular flow patterns were conducted on a wide scale. A considerable reduction of wave amplitude was noted on the interfacial surface as a result of damping of pulsations of annular flow by the very viscous liquid.

It was additionally indicated that an increase in the velocity of gas phase promotes the occurrence of higher waves on the surface of liquid film. With the exception of smooth films or light wavy ones these changes are irregular, which is manifested by the varied development of the interfacial surface. The occurrence of waves on the surface of phase separation is characterized by amplitude and length that are variable in time and space, which considerable complicated the quantitative description of the hydrodynamics of such phenomena.

From the analysis of experimental data one can conclude that within the entire range of variable process parameters all of them have a considerable effect on the formation of liquid film thickness. An increase in liquid viscosity always promotes an increase of mean thickness of liquid film. Concurrently, an increase in the volume flux of the gas phase for a constant equivalent Reynolds number for the liquid density leads to a thinner liquid film. Theses conditions are often accompanied by phenomena of wave interference, which promotes their accumulation or results in formation of additional capillary waves, which can lead to the occurrence of locally greater thickness.

\section{References}

Czernek, K. and Witczak, S. (2013) Non-invasive evaluation of wavy liquid film. Chemical and Process Engineering, 34, pp. 241-252.

Czernek, K. (2013) Hydrodynamic aspects design of thin film apparatuses for very viscous liquid, Opole University of Technology, Opole.

Lee, Y. J. and Kim, J. H. (1986) A review of holography applications in multiphase flow visualisation study, Journal of Fluids Engineering, 108, 3, pp. 279-289.

Oriol, J., Leclerc, J. P., Jallut, C., Tochon, P. and Clement, P. (2008) Characterization of the two-phase flow regimes and liquid dispersion in horizontal and vertical tubes by using coloured tracer and non-intrusive optical detector, Chemical Engineering Science, 63, 1, pp. 24-34.

Stahl, P. and Rohr, P. R. (2004) On the accuracy of void fraction measurements by single-beam gammadensitometry for gas-liquid two-phase flows in pipes, Experimental Thermal and Fluid Science, 28, 6, pp. 533544.

Troniewski, L., Witczak, S. and Czernek, K. (2006) Hydrodynamics and heat transfer during two-phase gas-high viscous liquid flow in film reactor, Chemical and Process Engineering, 27, pp. 1341-1359.

Wojtan, L., Ursenbacher, T. and Thome, J. R. (2005) Measurement of dynamic void fractions in stratified types of flow, Experimental Thermal and Fluid Science, 29, 3, pp. 383-392.

Xu, X. X. (2007) Study on oil-water two-phase flow in horizontal pipelines, Journal of Petroleum Science and Engineering, 59, 1-2, pp. 43-58. 